\title{
Spinal tuberculosis paraplegia associated with anterior spinal artery infarction: a very rare presentation.
}

\author{
Thillainathan S, Adikari M, Marasinghe IUK, Priyangika DKD, Premawansa G
}

\section{Introduction}

Tuberculosis remains a major cause of morbidity and mortality in developing countries. Paraplegia is the most dreaded complication of spinal tuberculosis. Here we present a patient with a clinical picture consistent with spinal tuberculosis associated with anterior spinal arterial infarction presenting as paraplegia.

\section{Case report}

A forty five year old lady from Colombo presented with subacute onset paraplegia with sensory level up to T8. She was apparently well until two months back when she developed cough which was not productive and not associated with fever for which she received some oral antibiotics. Over the next two weeks she developed severe backache localized to thoracic region, which was aggravated by movements but not radiating to legs. There was no associated spinal deformity or kyphosis. Subsequently she noticed weakness and numbness of bilateral lower limbs which developed within two days duration while upper limbs were preserved. Same time she developed urinary and faecal incontinence. Examination revealed sensory level of T6 with impaired pain and temperature sensation below T6 level with exaggerated deep reflexes of lower limbs. Joint position sensation and vibration were intact.

Initially transverse myelitis was suspected on clinical grounds and intravenous immunoglobulin therapy was given along with intravenous dexamethasone for five days. There was some improvement in paraplegia but it was not sustained. A gadolinium-enhanced MRI of the brain excluded multiple sclerosis which could be associated with transverse myelitis. Urgent magnetic resonance imaging (MRI) of brain and whole spine revealed bilateral homogenously enlarged lacrimal glands, a soft tissue density in anterior epidural space extending from C8/T1 to T6/T7 level with mild spinal cord oedema. MRI and High-resolution computed tomography chest showed a soft tissue density lesion in left hilar region with multiple nodular opacities scattered in both lung fields. There was no mediastinal or hilar lymphadenopathy. (Figure 1)

General Medical Unit, Colombo North Teaching Hospital, Ragama.

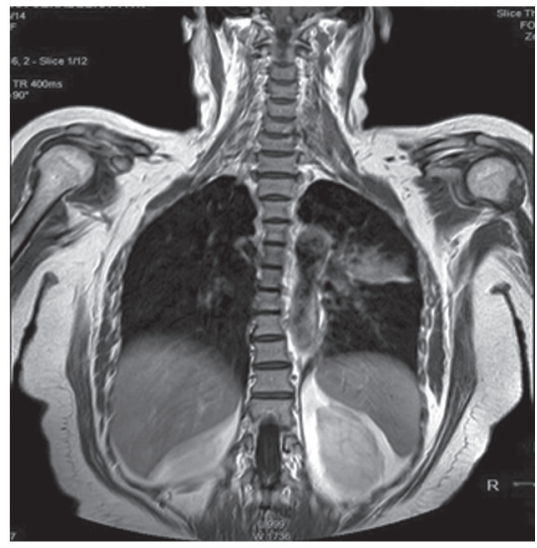

Figure 1: MRI scan of chest revealing a soft tissue density lesion in left hilar region (white arrow) with multiple nodular opacities.

Paraplegia associated with dorsal spine anterior epidural soft tissue density as well as the hilar mass raised the suspicion of lymphoma or tuberculosis while bilateral enlarged lacrimal glands pointed towards sarcoidosis. Angiotensin-converting-enzyme inhibitor level and serum calcium level were persistently low. She underwent vedio-assisted thoracoscopy and biopsy was taken from the hilar mass. Interestingly microscopy of the lung tissue revealed multiple and large foci of granulomas composed of aggregates of epitheloid histiocytes, langerhans type multinucleated giant cells and central caseation. Ziehl-Neelsen stain showed fragmented acid fast bacilli and the diagnosis of caseous tuberculosis was strongly suggested.

Erythrocytic sedimentation rate was $75 \mathrm{~mm}$, sputum for acid fast bacilli, sputum culture and mantoux test were negative. She was started on anti-tuberculosis treatment (ATT) with isoniazid, rifmpicin, pyrazinamide and ethambutol. On day 10 she developed severe drug induced hepatitis leading to hepatic encephalopathy and was admitted to the intensive care unit for observation. Once she became clinically and biochemically stable, under very close observation ATT regime was reintroduced gradually and was tolerated.

During the treatment process MRI whole spine was repeated ten weeks later which, to our curiosity, showed clearing of the previously noted epidural soft tissue density revealing a low intensity lesion in T1 weighted images and a high signal intensity lesion in T2 weighted images indicating a short segment anterior spinal artery territory infarction from T3 to T5 level (Figure 2.3). The 
lesion was darker than the gray matter in contrast to the substance of cord.

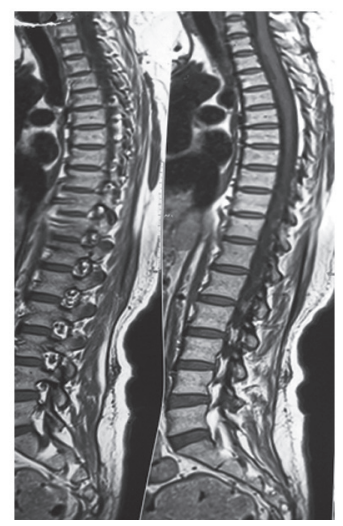

Figure 2: $T 1$ weighted MRI scan of spine displays low signal intensity from T3 to T5 level (Black arrow) in sagittal view.

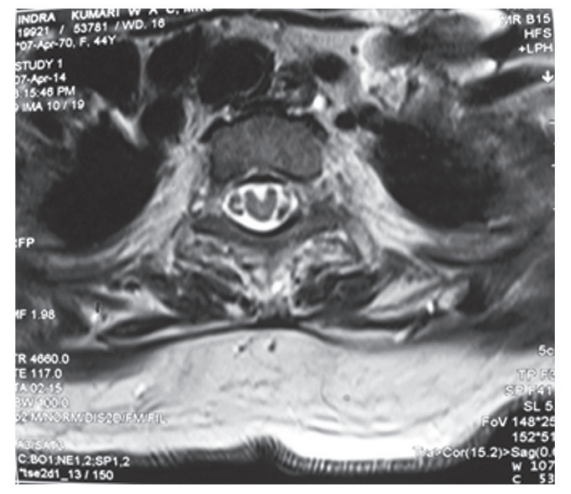

Figure 3: T2 weighted MRI scan displays high signal intensity lesion (Black arrow) in the anterior part of the spinal cord.

Thrombophilic screening proved negative. 2D Echocardiogram excluded any cardiac source of embolism. Tuberculosis endarteritis leading to the obliteration of the anterior spinal artery which is a rare cause for spinal tuberculosis paraplegia was diagnosed. With antituberculosis treatment and continuous physiotherapy gradually she gained some sustained improvement of being able to move the legs but movement of lower limbs against gravity was not achieved and sensory impairment and incontinence persisted.

\section{Discussion}

Around $10 \%$ of patients with extra pulmonary tuberculosis may have bone and joint involvement and half of them will have tuberculosis of spine. [1] Among spinal tuberculosis the incidence of neurological involvement is $10-46 \%$. [2] Spinal tuberculosis is common among children and young adolescents but may occur in any age group. Paraplegia is the most dreaded complication of spinal tuberculosis.
Among the patients with active spinal tuberculosis, paraplegia may be caused by direct involvement of meninges and spinal cord by the tuberculous infection or by direct mechanical pressure on the spinal cord by tubercular abscess, caseous granulation tissue and debris or by intrinsic changes in the spinal cord such as inflammatory oedema. Infective thrombosis or endarteritis of spinal vessels leading to infarction of the spinal cord is a very rare cause of paraplegia. [3]

Granulomatous endarteritis of anterior spinal artery is a rare specific manifestation of spinal tuberculosis. In our patient the infection seemed to have spread from the hilar caseous lesion via haematological seeding to the arteries of spinal cord. Infarction of spinal cord is an unusual but important cause of paraplegia and it is caused by endarteritis, periarteritis or thrombosis of an important tributary to the anterior spinal artery caused by inflammatory reaction [4].

Spiller WG (1909) first described anterior spinal artery syndrome as having pain at the level of lesion disturbance of pain and temperature, paraparesis or tetraparesis and urinary incontinence [5]. Currently MRI is considered as the best modality of choice for confirming the diagnosis of anterior spinal artery syndrome in suspected patients. [6] Relative to normal spinal cord, the lesions of anterior spinal artery infarction appear hyperintense in T2 weighted images while it appears as hypointense lesions in T1 weighted images and the lesions are usually seen anteriorly in spinal cord. [6] A finding of vertebral body infarction contiguous to a cord signal abnormality on $\mathrm{MRI}$ is a definite indicator of ischemia and a useful confirmatory sign if present; however, this is established in only 4 to 35 percent of patients, and its absence does not exclude spinal cord infarction. [7]

We believe this patient suffered from an infraction of the spinal cord because of the typical clinical features on presentation, absence of cord compressions, and exclusion of other known neurological diseases. A typical loss of motor neuron function with dissociated sensory impairment below the level of the lesion pointed to anterior spinal artery syndrome [8] as the result of an infraction occurring in the region supplied by this artery. $\mathrm{MRI}$ during the acute stage revealed soft tissue intensity in anterior epidural space with mild spinal cord oedema. Ten weeks later repeat MRI revealed a low intensity lesion in T1 weighted images and a high signal intensity lesion in T2 weighted images indicating distinct focus of short segment anterior spinal artery territory infarction from T3 to T5 level. A similar finding was reported in patients with spinal cord ischemia. [9]

This unique case emphasizes that obliterative tuberculous endarteritis leading to anterior spinal artery occlusion should be considered in the differential diagnosis of any 
paraplegia, especially in countries with a high incidence and prevalence of tuberculosis.

\section{References}

1. Gautam MP, Karki P, Rijal S, et al. Potts spine and Potts paraplegia. J Nep Med Assoc 2005; 44 (159):106-15.

2. Omari B, Robertson JM, Nelson RJ, et al. Pott's disease: a resurgent challenge to the thoracic surgeon. Chest 1989; 95:145-50.

3. Dunn R, Zondagh I, Candy S. Spinal Tuberculosis. Magnetic Resonance Imaging and Neurological Impairment. SPINE 2011; 36(6): 469-73.

4. Tuli SM. Tuberculosis of the skeletal system (bones, joints, spine and bursal sheaths). Second Ed. New Delhi: Jaypee Brothers Medical Publishers (P) Ltd, 1991:3-122.
5. Spiller WG. Thrombosis of the cervical anterior median artery; syphilitic acute anterior poliomyelitis. J Nerv Ment Dis 1909;36:601-13.

6. Takahashi S, Yamada T, Ishii K, et al. MRI of anterior spinal artery syndrome of the cervical spinal cord. Neuroradiology 1992;35:25-9

7. Cheng MY, Lyu RK, Chang YJ, et al. Concomitant spinal cord and vertebral body infarction is highly associated with aortic pathology: a clinical and magnetic resonance imaging study. J Neurol 2009; 256:1418-26.

8. Foo $D$, Rossier $A B$. Anterior spinal artery syndrome and its natural history. Paraplegia 1983; 21:1-10.

9. Kume A, Yoneyama S, Takahashi A, Watanabe H. MRI of anterior spinal artery syndrome. J Neurol Neurosurg Psychiatry 1992;55:838-40. 